\title{
Electric ion dispersion as a new type of mass spectrometer
}

\author{
Michael Lindstrom ${ }^{1 *}+$, lain Moyles $^{2+}$ and Kevin Ryczko ${ }^{3}$
}

\author{
*Correspondence: \\ mikel@math.ucla.edu \\ ${ }^{\dagger}$ Equal Contributor \\ 1 University of California, Los \\ Angeles, USA \\ Full list of author information is \\ available at the end of the article
}

\begin{abstract}
At the 2014 Fields-MPrime Industrial Problem Solving Workshop, PerkinElmer presented a design problem for mass spectrometry. Traditionally, mass spectrometry is done via three methods: using magnetic fields to deflect charged particles whereby different masses bend differently; using a time-of-flight procedure where particles of different mass arrive at different times at a target; and using an electric quadrupole that filters out all masses except for one very narrow band. The challenge posed in the problem was to come up with a new design for mass spectrometry that did not involve magnetic fields and where mass fractions could be measured in an entire sample on a continuous basis. We found that by sending the sample particles down a channel of line charges oscillations would be induced with a spatial wavelength being mass dependent thereby allowing different masses to be separated spatially and potentially detected on a continuous basis, without the use of magnetic fields. In this paper, we present the analysis of our design and illustrate how this principle could be used for mass spectrometry.
\end{abstract}

Keywords: Mass spectrometry, Electric fields, Mass dispersion, Harmonic motion

\section{Introduction}

\section{Mass spectrometry}

Mass spectrometry is a technique used to determine the chemical composition of a certain substance or substances by separating atomic elements by mass. The most common underlying mechanism of most mass spectrometers is by ionizing each particle, giving each particle the same net positive charge and exploiting their mass-charge ratio. There are three mechanisms that are primarily used for industrial mass spectrometers [4]. One technique uses magnetic fields to separate masses into rings of distinct radii which can then be detected. The use of magnetic fields, however, is quite costly and was not desired by our industrial partner. The second most common technique is a procedure known as time of flight which excites the mass sample by giving it kinetic energy and sending the particles through a given path. Because the particles will have different accelerations, this makes all the particles have different velocities, and hence, for a fixed detector position, they will have different travel times. It was indicated to us by the industrial partner, however, that this method is also not desirable because the kinetic excitation comes in pulses of energy which means that any transient changes in chemical composition will not be detected. With these considerations in mind, it was desired by the industrial sponsor to design a mass spectrometer that only uses electric fields and that does not lose any sample

(C) 2016 Lindstrom et al. Open Access This article is distributed under the terms of the Creative Commons Attribution 4.0 International License (http://creativecommons.org/licenses/by/4.0/), which permits unrestricted use, distribution, and reproduction in any medium, provided you give appropriate credit to the original author(s) and the source, provide a link to the Creative Commons license, and indicate if changes were made. 
resolution. It was also noted that devices that "trap" particles, confining them to localized spatial regions for later detection, are less desirable as this again does not allow for continuous measurement of composition.

Another device that is used is known as a quadrupole mass spectrometer (cf. $[2,4,5])$ which works as a bandpass filter separating masses. The design has four conductors which have a base $\mathrm{DC}$ voltage plus an $\mathrm{AC}$ voltage such that diametrically opposite electrodes have the same potential and adjacent electrodes have opposite voltages. The AC voltage is such that heavy masses do not react to the changing polarity fast enough, effectively only feeling the base DC and hence slowly drifting to the conductor that was originally negatively charged and eventually annihilating. This is the low-pass component of the filter. Conversely, the lighter positive charges feel the effect of the modulating AC and do not notice the DC. Very light charged particles are impacted by the AC very quickly, being excited due to resonance effects [5] and thereby collide with the electrodes near entry. This is the high-pass filter component of the device. With these effects combined, parameters can be chosen to isolate a band of a single mass to pass through the detector. In order to detect several masses, industrialists need to inject repeated copies of the sample and adjust parameters to change the isolated mass. While the industrial partner was generally happy with this device, they were concerned about the time taken to process individual masses as well as the potentially limited amount of initial sample available. In this paper, we explore the task of finding a device that could detect multiple masses at once keeping these stipulations in mind.

\section{Electric ion dispersion}

Our idea presented in this paper is based on harmonic oscillation. If two fixed positive point charges were separated and a positive charge were introduced at a point away from the equilibrium of the charges, oscillations about the equilibrium would occur in a one-dimensional system. The period of these oscillations will be mass dependent. Likewise, if the charged particles were deflected from the edges of a long device and all maintained a roughly constant axial velocity, the temporal periods will translate into spatial wavelengths whereby different masses have different wavelengths. Such a property could be exploited to induce a mass dispersion (see Fig. 1). Note that we consider a series of isolated point charges instead of a line of charge. In the limit, as we show, this point charge model reduces to a line charge model. It is potentially more practical from an engineering perspective to have isolated charges insulated from each other than line charges. By analyzing the model with these isolated charges, we in effect analyze the line charge model as well. Observe that this device operates in a plane. While it may seem natural to extend this to a solenoidal geometry, as we observe in our modelling work, a solenoid approximates a cylinder with constant boundary potential too well and thus the desired effect is not achieved as the net electric field inside is approximately zero.

\section{Methods}

In this paper, we consider two designs for mass dispersion devices. The first design illustrates a proof-of-principle for our ion dispersion technique and is two-dimensional, with the sides consisting of isolated charges along a line. This model will be amendable to mathematical analysis. The second design is less straightforward to analyze, and we rely 


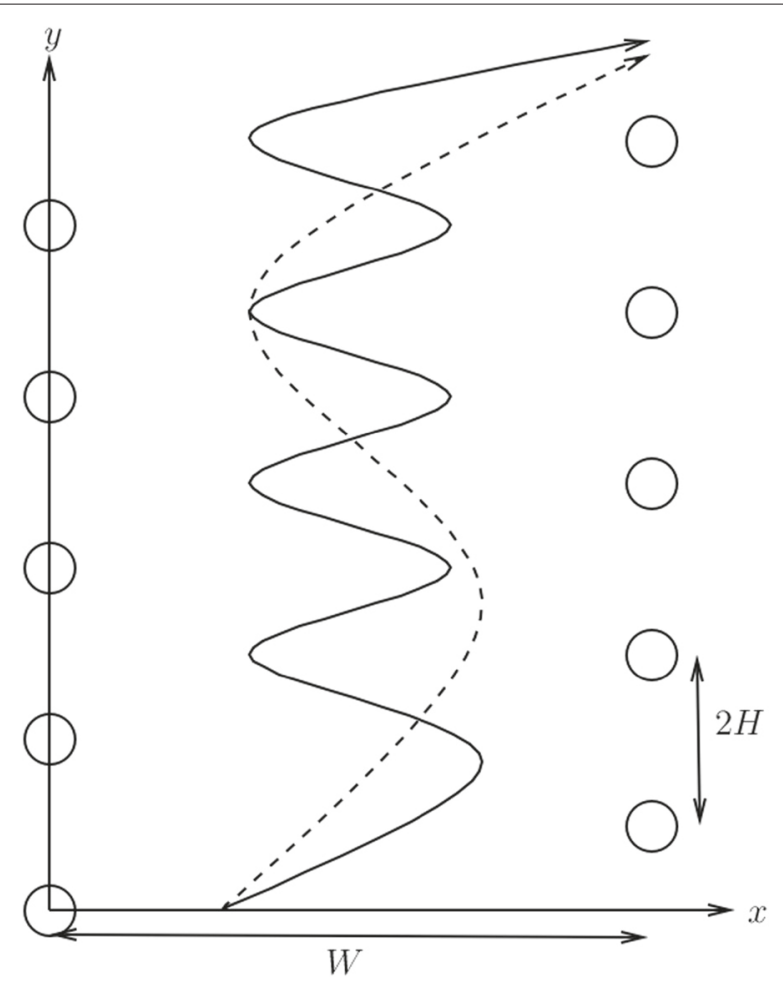

Fig. 1 A sketch of the line charge device: within the plane, particles enter from the bottom and are excited into oscillations in $x$ with approximately constant $y$-velocity. The circles indicate positive charges of charge $+Z$ e with a vertical spacing of $2 \mathrm{H}$. In the implementation, likely $\mathrm{H}$ would be very small so that particles cannot exit through the sides of the device or, if practical considerations are required, the isolated charges could be replaced by a line of charges with uniform linear charge density

upon numerical methods. It consists of a helical wire carrying a uniform linear charge density. It is unfortunately not useful in producing the desired mass dispersion effects. All of our analysis is based on simple principles of non-relativistic electrostatics, for which [3] is an excellent reference.

\section{Line charge design formulation}

Charges with net charge $Z e$ are placed along the line $x=0$ at positions $y=$ $0,2 H, 4 H, 6 H, \ldots$ and along the line $x=W$ at positions $y=H, 3 H, 5 H, 7 H, \ldots$. The staggering adds an extra level of protection that the moving particles do not escape through the sides of the device. Any charges that are placed in the dispersion device will experience forces from the fixed point charges along the device via Coulomb's law:

$$
\begin{aligned}
V & =\frac{Z e}{4 \pi \epsilon_{0}} \sum_{j=1}^{N}\left(\frac{1}{\sqrt{x^{2}+(y-2 j H)^{2}}}+\frac{1}{\sqrt{(x-W)^{2}+(y-(2 j-1) H)^{2}}}\right), \\
m \ddot{x} & =-e \nabla V
\end{aligned}
$$

where we neglect particle-particle interactions at this point. Here, $e$ is the fundamental electric charge, $Z$ is the charge number for the fixed point charges on the device, $N$ is the half the number of device charges on each side of the device, $H$ is the half the vertical spacing between adjacent charges (due to the non-symmetric staggered charge distribution 
across the two sides of the device), $\epsilon_{0}$ is the permeativity of free space, and $m$ is the mass of the entering test charge. We make the following non-dimensionalizations:

$$
x=W \bar{x}, \quad y=W \bar{y}, \quad t=\frac{W}{U_{0}} \bar{t}
$$

where $W$ is the width of the device and $U_{0}$ is the magnitude of a typical incoming particle velocity, i.e.,

$$
U_{0}=\sqrt{U_{0 x}^{2}+U_{0 y}^{2}} .
$$

If we define the following quantities

$$
\begin{aligned}
h & =\frac{H}{W}, \\
\beta_{m} & =\frac{Z e^{2} m_{p} / m}{4 \pi \epsilon_{0} m_{p} W U_{0}^{2}},
\end{aligned}
$$

then we can write our non-dimensional system as (dropping the overbars for convenience)

$$
\begin{aligned}
& \ddot{x}=\beta_{m} \sum_{j=1}^{N}\left(\frac{x}{r_{j}^{3}}+\frac{x-1}{\rho_{j}^{3}}\right), \\
& \ddot{y}=\beta_{m} \sum_{j=1}^{N}\left(\frac{y-2 j h}{r_{j}^{3}}+\frac{y-(2 j-1) h}{\rho_{j}^{3}}\right)
\end{aligned}
$$

where

$$
\begin{aligned}
r_{j} & =\sqrt{x^{2}+(y-2 j h)^{2}}, \\
\rho_{j} & =\sqrt{(x-1)^{2}+(y-(2 j-1) h)^{2}} .
\end{aligned}
$$

All parameter values are listed in Table 1.

\section{Initializations}

\section{Design initial conditions}

Given $x=y=0$ is the bottom-left corner of the line charge device, then in order to have incoming particles enter from outside the device, we require

$$
y(0)=y_{0}<0 .
$$

Table 1 Parameters used in non-dimensional model of ion dispersion

\begin{tabular}{lll}
\hline Parameter & Description & Value \\
\hline$W$ & Width of device & $0.1 \mathrm{~m}$ \\
$H$ & Half charge spacing on device & $1 \times 10^{-4} \mathrm{~m}$ \\
$U_{0}$ & Incoming particle velocity & $\sqrt{2} \times 10^{4} \mathrm{~ms}^{-1}$ \\
$e$ & Elementary charge constant & $1.6 \times 10^{-19} \mathrm{C}$ \\
$Z$ & Charge number of device charges & 15 \\
$N$ & Quantity of device charges on each side of device & 20,000 \\
$\epsilon_{0}$ & Permeativity of free space & $8.85 \times 10^{-12} \mathrm{CV}^{-1} \mathrm{~m}^{-1}$ \\
$m_{p}$ & Mass of a proton & $1.6726 \times 10^{-27} \mathrm{~kg}$ \\
\hline
\end{tabular}


It was previously mentioned that the premise of the device is oscillation around the equilibrium of point charges. This equilibrium lies directly at the center of the device and therefore, in order to have periodic motion, we take

$$
x(0)=x_{0}, \quad 0<x_{0}<\frac{1}{2} .
$$

As we do not need an initial horizontal velocity, we can set

$$
\dot{x}(0)=0
$$

because by placing the particle away from equilibrium with respect to the device charges, we will induce horizontal motion for $t>0$. Imposing the initial vertical velocity is slightly less straightforward. If the velocity is too high, then the particles will enter and exit the device before any effective horizontal motion can begin. Conversely, if the charge is too small, then the incoming particle will be repelled by the charges in front of it and will not have enough energy to enter the device. We therefore need the velocity to be sufficient to overcome the initial potential barrier but to not be too large. To approximate the potential barrier, we consider a "center of charge" argument where all of the wall charges (2NZ total) are concentrated as a single charge in the center of the device $(1 / 2, N H)$. If this were the case, we want to give the particle enough kinetic energy $E_{v}=1 / 2 m \dot{y}(0)^{2}$ to overcome the potential difference of its relative starting position to the group charge

$$
E_{0}=\frac{2 N Z e^{2}}{4 \pi \epsilon_{0}} \frac{1}{\sqrt{\left(x_{0}-W / 2\right)^{2}+\left(N H-y_{0}\right)^{2}}}
$$

and to the half-distance between the device entrance and the group charge at the center,

$$
E_{1}=\frac{2 N Z e^{2}}{4 \pi \epsilon_{0}} \frac{1}{\sqrt{\left(x_{0}-W / 2\right)^{2}+(N H / 2)^{2}}} .
$$

Using our non-dimensional scaling, we get the following inequality for the vertical velocity,

$$
\dot{y}(0)>\sqrt{\frac{4 \beta_{m} N}{\left(\left(x_{0}-\frac{1}{2}\right)^{2}+(N h)^{2}\right)^{1 / 2}}-\frac{4 \beta_{m} N}{\left(\left(x_{0}-\frac{1}{2}\right)^{2}+\left(y_{0}-N h\right)^{2}\right)^{1 / 2}}} .
$$

Using the values $x_{0}=1 / 4$ and $y_{0}=-1$ along with the parameters in Table 1 , we get that

$$
\dot{y}(0) \gtrsim 0.02 \text {, }
$$

i.e., the initial velocity has to be at least $2 \%$ of the dimensional $U_{0}$ velocity. To avoid stalling inside the device, we will use a value $\dot{y}(0)=0.2$. According to our industrial partner, this velocity is easy to achieve for any initial concentration. Our argument for the initial velocity for the solenoidal design is identical.

\section{Solenoid device}

We now briefly present the second design where we consider a solenoidal geometry with a wire of uniform charge density parameterized as a helical segment. Our wire takes the form

$$
\langle x(\theta), y(\theta), z(\theta)\rangle=\left\langle R_{0} \cos (\theta), R_{0} \sin (\theta), \alpha R_{0} \theta\right\rangle
$$

with $0 \leq \theta \leq \frac{2 N H}{R_{0} \alpha}$, carrying a uniform charge density

$$
\lambda=\frac{Z e}{2 \pi R_{0} \sqrt{1+\alpha^{2}}} .
$$


By setting $R_{0}=W / 2$, this solenoid has the same width as the separated charges and with

$$
\alpha=\frac{H}{2 \pi R_{0}}
$$

the spacing between charges in the separated charge design corresponds to the $z$-distance between consecutive helical rings. Also, for every change of $\theta$ by $2 \pi$, the wire length is $2 \pi R_{0} \sqrt{1+\alpha^{2}}$ giving a total charge of Ze. With this, using Eq. (4), the potential energy of a particle of charge $e$ is

$$
V=\frac{Z e}{8 \pi^{2} \epsilon_{0}} \int_{0}^{\frac{2 N H}{R_{0} \alpha}} \frac{\mathrm{d} \theta}{\sqrt{\left(x-R_{0} \cos \theta\right)^{2}+\left(y-R_{0} \sin \theta\right)^{2}+\left(z-R_{0} \alpha \theta\right)^{2}}} .
$$

This integral is obtained by integrating the potential energy contribution of each differential arc length element of the helix over the length of the helix.

We then make the non-dimensionalizations:

$$
\langle x, y, z\rangle=R_{0}\langle\bar{x}, \bar{y}, \bar{z}\rangle, \quad t=\frac{R_{0}}{U_{0}} \bar{t}
$$

and define

$$
\gamma_{m}=\frac{Z e^{2} m_{p} / m}{8 \pi^{2} \epsilon_{0} m_{p} R_{0} U_{0}^{2} \sqrt{1+\frac{H^{2}}{4 \pi^{2} R_{0}^{2}}}} .
$$

In dimensionless form, the equations of motion $m \frac{\mathrm{d}^{2}}{\mathrm{~d} t^{2}}\langle x, y, z\rangle=-e \nabla V$, again dropping the overbars, can be expressed by

$$
\langle\ddot{x}, \ddot{y}, \ddot{z}\rangle=\gamma_{m} \int_{0}^{\frac{2 N H}{R_{0} \alpha}} \frac{\langle\cos \theta-x, \sin \theta-y, \alpha \theta-z\rangle}{\left((x-\cos \theta)^{2}+(y-\sin \theta)^{2}+(z-\alpha \theta)^{2}\right)^{3 / 2}} \mathrm{~d} \theta
$$

\section{Results and Discussion}

\section{Line charge numerical results}

We implemented the model using ode 45 in Matlab choosing parameters from Table 1 and taking $x(0)=1 / 4, y(0)=-1, \dot{x}(0)=0$, and $\dot{y}(0)=0.2$. Figure 2 shows the $x-y$ trajectories for a series of masses $\left(0.5 m_{p}, m_{p}\right.$, and $\left.2 m_{p}\right)$ with the blue curve being the trajectory for mass $0.5 m$, the black curve for mass $m$, and the red curve for mass $2 m$. The spacing between charges is non-dimensionally $h$, and so, the length of the device is $2 N h$.

Very quickly, we see that the mass dispersion relation has separated the three particles. Figure 3 shows the $y$-velocity for the mass $m$.

While there is a vertical acceleration through the device, it is quite small. This effect is seen in any mass that enters the device with smaller masses having the larger accelerations. Therefore, the device appears to have the original intention of maintaining $y$-velocity and reducing the problem to that of a single oscillator between two charges. In the sections that follow, comparisons with numerics refer to plots generated using this ode4 5 implementation.

\section{A posteriori justification of negligible interaction forces}

Although we neglected the interaction between individual charged particles in the numerical results, focusing solely upon the forces induced by the charged cavity on the particles, 


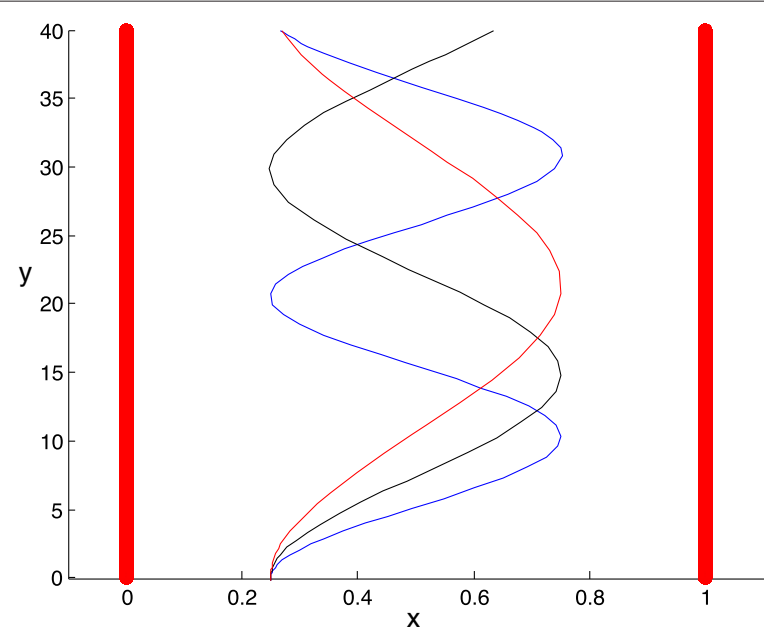

Fig. 2 The red lines along the side represent the small-spaced point charges while the curves inside are the trajectories of different masses. The blue curve is the trajectory for the mass $0.5 \mathrm{~m}$, the black curve is the trajectory for mass $m$, and the red curve is the trajectory for mass $2 m$

a

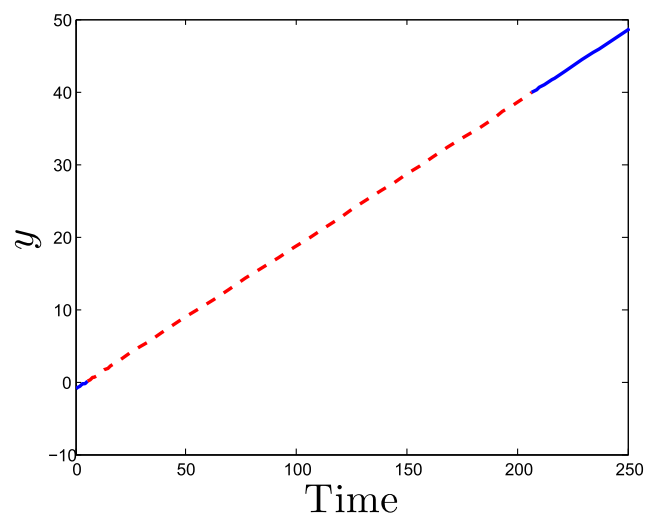

b

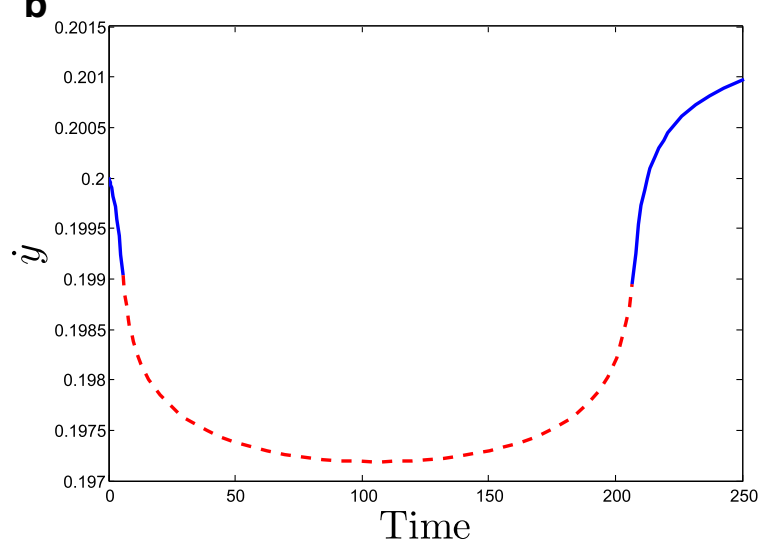

Fig. 3 a, b Plot of $y$-position (Fig $\mathbf{a}$ ) and $y$-velocity (Fig $\mathbf{b}$ ) over time for particle of mass $m=m_{p}$. The "sharp" transition regions are where the particles enter and exit the device separating two regions of essentially constant velocity. The transition regions themselves experience a very small acceleration. The solid blue curves represent the particle being outside of the device while the red dashed curve represents the particle inside of the device 
we justify the negligible forces by considering how big the force between two particles would be if they traveled along their natural trajectories $\left(x_{1}(t), y_{1}(t)\right)$ and $\left(x_{2}(t), y_{2}(t)\right)$ by considering only the forces induced by the charged walls. We consider two particles of dimensionless mass $1 m_{p}$ and $2 m_{p}$, originating at $(1 / 4,-1)$ with zero initial $x$-velocity and a $y$-velocity of 0.2 , and plot the force

$$
f(t)=\frac{\tilde{\beta}_{m} m / m_{p}}{\left(x_{1}(t)-x_{2}(t)\right)^{2}+\left(y_{1}(t)-y_{2}(t)\right)^{2}}
$$

versus time, both in dimensionless units, when both particles have entered the device. Note that $\tilde{\beta_{m}}=\beta_{m} / 15$ because the charge of the individual particles is $Z=e$ and $\operatorname{not} Z=$ $15 e$. We also can compute the dimensionless force of the device acting on the particles over that same range of time via

$$
f_{j}(t)=a_{j}(t) / \beta_{m}
$$

where $a_{j}(t)$ is the magnitude of the dimensionless acceleration of particle $j$.

As we observe in Figs. 4 and 5, the dimensionless force exerted on the particles by the device greatly exceeds the force exerted between the two particles on each other by several orders of magnitude. The smallest force exerted on the particles by the device is still roughly 60 and generally much larger, whereas the typical scale of the force between the two particles is $1 \times 10^{-4}$. The plots of the forces are for two particles, but even for hundreds, the forces between particles would be negligible. Particle collisions are another possibility, but these tend to be very infrequent and the main effect would be to reduce the particle velocities as noted in [1].

\section{Line charge analytic results}

Since $N$, the number of charges on each side of the device, is so large in the staggered charge model, we expect that the summation in Eq. (2) can be replaced by an integral. Indeed, this is how a line charge model can be derived by taking a limit of point charges. The device sides in Fig. 2 are discrete charges but appear as a single line charge when

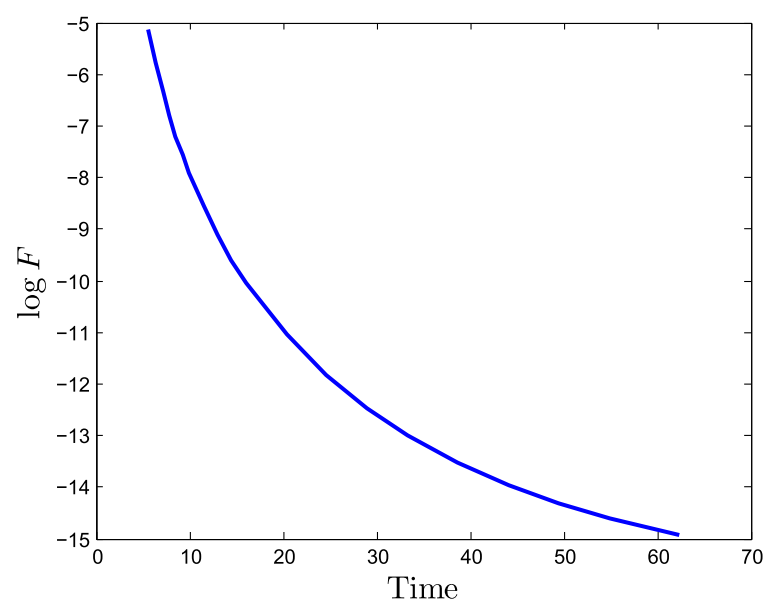

Fig. 4 The log of the force $F$ between the two particles of mass $m_{p}$ and $2 m_{p}$ as they move along their trajectories 


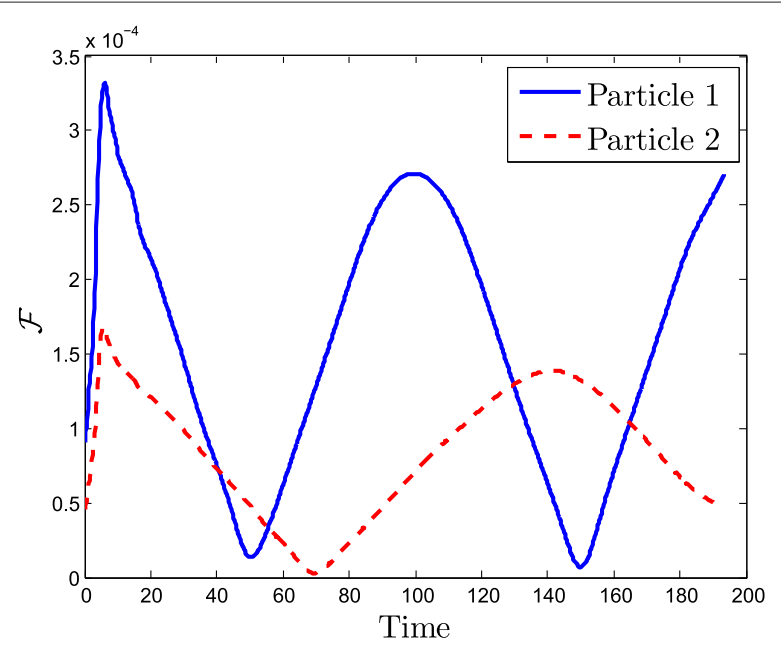

Fig. 5 The dimensionless force exerted by the device on the particles of mass $m_{p}$ and $2 m_{p}$ denoted by $\mathcal{F}$

the spacing is very small. Performing a Riemann summation, we can approximate the following sums as integrals:

$$
\begin{gathered}
\beta \sum_{j=1}^{N} \frac{x}{r_{j}^{3}} \approx-\frac{\beta}{2 h} \int_{y}^{y-2 N h} \frac{x}{\left(x^{2}+s^{2}\right)^{3 / 2}} \mathrm{~d} s \\
\beta \sum_{j=1}^{N} \frac{y-2 j h}{r_{j}^{3}} \approx-\frac{\beta}{2 h} \int_{y}^{y-2 N h} \frac{t}{\left(x^{2}+q^{2}\right)^{3 / 2}} \mathrm{~d} q .
\end{gathered}
$$

Similar integration transformations follow for the $\rho_{j}$ summations. Using these integrals as replacements in Eq. (2) and performing the integration, we get a new line charge continuum model:

$$
\begin{aligned}
& \ddot{x}=-\frac{\beta}{2 h x}\left(\frac{y-2 N h}{\rho_{x, y-2 N h}}-\frac{y}{\rho_{x, y}}\right)-\frac{\beta}{2 h(x-1)}\left(\frac{y-2 N h}{\rho_{x-1, y-2 N h}}-\frac{y}{\rho_{x-1, y}}\right) \\
& \ddot{y}=-\frac{\beta}{2 h}\left(\frac{1}{\rho_{x, y}}-\frac{1}{\rho_{x, y-2 N h}}+\frac{1}{\rho_{x-1, y}}-\frac{1}{\rho_{x-1, y-2 N h}}\right)
\end{aligned}
$$

where

$$
\rho_{a, b}=\sqrt{a^{2}+b^{2}}
$$

Solving (Eq. 7) numerically using ode45 and comparing it to the numeric computations of Eq. (2) for a single mass $m$ is displayed in Fig. 6 which shows relatively strong agreement.

In the design of interest, the length $L=2 N h \gg 1$ and we can exploit this to obtain a leading-order asymptotic estimate for the accelerations given in Eqs. (7a) and (7b) when $0<x<1$ and $y=L / 2+u$ with $|u| \ll L$, i.e., when the particles are well into the device with respect to either end. In essence, we are performing an 


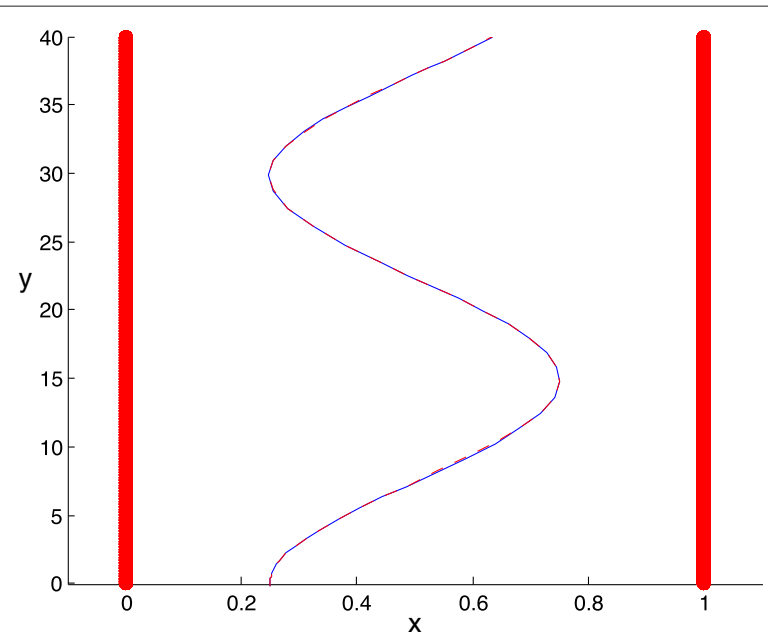

Fig. 6 The trajectories of a single mass $m$ with the blue solid curve representing the trajectory for the full summation model (Eq. 2) and the red dashed curve representing the trajectory for the model (Eq. 7) with the summations replaced by integrals

asymptotic expansion in the acceleration equations starting at some time when $y \approx N h$. We write

$$
\begin{aligned}
\ddot{x}= & -\frac{\beta}{2 h x}\left(\frac{u-L / 2}{\sqrt{x^{2}+(u-L / 2)^{2}}}-\frac{L / 2+u}{\sqrt{x^{2}+(u+L / 2)^{2}}}\right) \\
& -\frac{\beta-L / 2}{2 h(x-1)}\left(\frac{u}{\sqrt{(x-1)^{2}+(u-L / 2)^{2}}}-\frac{u+L / 2}{\sqrt{(x-1)^{2}+(u+L / 2)^{2}}}\right) \\
= & -\frac{\beta}{2 h x}\left(\frac{-L / 2+o(L)}{\sqrt{L^{2} / 4+O(L)}}-\frac{L / 2+o(L)}{\sqrt{L^{2} / 4+O(L)}}\right) \\
& -\frac{\beta}{2 h(x-1)}\left(\frac{-L / 2+o(L)}{\sqrt{L^{2} / 4+O(L)}}-\frac{L / 2+o(L)}{\sqrt{L^{2} / 4+O(L)}}\right) \\
& \sim \frac{-\beta}{h x}+\frac{\beta}{h(x-1)}+\mathcal{O}\left(1 / L^{2}\right)
\end{aligned}
$$

and

$$
\begin{aligned}
\ddot{y}= & -\frac{\beta}{2 h}\left(\frac{1}{\sqrt{x^{2}+(L / 2+u)^{2}}}-\frac{1}{\sqrt{x^{2}+(u-L / 2)^{2}}}+\frac{1}{\sqrt{(x-1)^{2}+(L / 2+u)^{2}}}\right) \\
& \left.-\frac{1}{\sqrt{(x-1)^{2}+(u-L / 2)^{2}}}\right) \\
= & -\frac{\beta}{2 h}\left(\frac{2}{L}-\frac{2}{L}+\frac{2}{L}-\frac{2}{L}\right)-\frac{8 \beta u}{h L^{2}}+\mathcal{O}\left(1 / L^{4}\right) \\
& \sim-\frac{8 \beta u}{h L^{2}} .
\end{aligned}
$$

From this asymptotic work taking terms of size $\mathcal{O}(1)$, we consider far simpler acceleration equations for our analytic work, namely

$$
\ddot{x}=\frac{\beta}{h}\left(\frac{1}{x}+\frac{1}{x-1}\right)
$$


and

$$
\ddot{y}=0 .
$$

This nearly constant $y$-velocity as mentioned in the numerical results is expressed by the zero $y$-acceleration.

If we consider Eq. (9) a final simplification of our whole model, then we can actually use it to approximate the period of oscillation. Firstly, we can multiply (Eq. 9) by $\dot{x}$ and integrate to get

$$
\dot{x}= \pm \sqrt{\frac{2 \beta}{h}} \log \left(\frac{x(x-1)}{x_{0}\left(1-x_{0}\right)}\right)
$$

where the change in sign comes from the transition through turning points in the oscillation. Note that this model is only valid near the middle of the device and this $x_{0}$ value is the initial position away from equilibrium for the trajectory in this asymptotic regime where $x_{0}<1 / 2$ and $\dot{x}=0$ when $x=x_{0}$; it is not necessarily the same as the $x_{0}$ value in the initializations for the numerics. If we integrate this expression once more from the initial time $t=0$ where $x=x_{0}$ to the turning time $t=T_{1 / 2}$ where $x=1-x_{0}$, then we recover the half-period,

$$
T_{1 / 2}=\sqrt{\frac{2 h}{\beta}} I\left(x_{0}\right)
$$

where

$$
I\left(x_{0}\right)=\int_{1 / 2}^{1-x_{0}} \frac{\mathrm{d} x}{\sqrt{\log (x(1-x))-\log \left(x_{0}\left(1-x_{0}\right)\right)}} .
$$

Here we integrate only from the equilibrium position by exploiting an even symmetry in the integral. Given a fixed initial position, $I\left(x_{0}\right)$ can be computed numerically. The integral is singular but integrable. If all parameters are fixed aside from mass, then since $\beta$ depends inversely on mass we have a dispersion relation,

$$
T_{1 / 2} \propto \sqrt{m} .
$$

We plot the full period ( $T=2 T_{1 / 2}$ ) dispersion relation in Fig. 7 with the parameters taken from Table 1 and the simulations in the "Line charge numerical results" section.

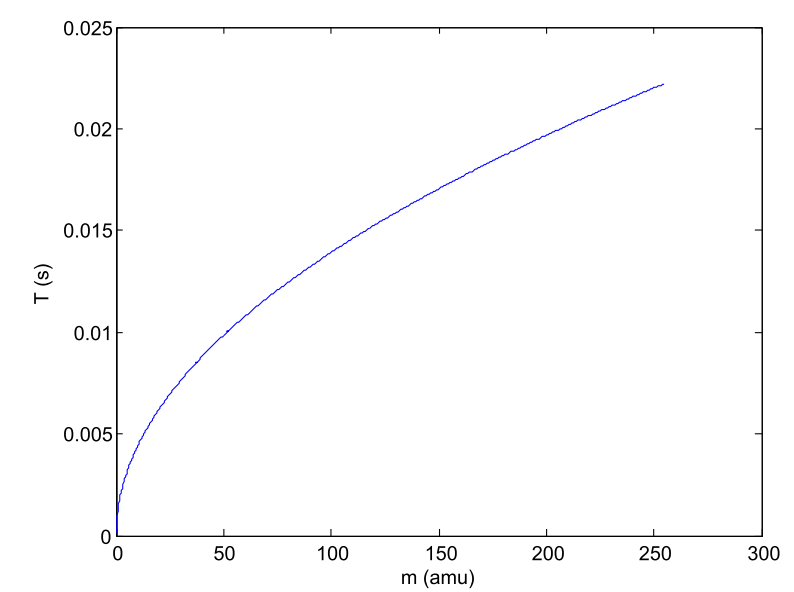

Fig. 7 Dispersion relation of the period $T$ versus the mass $m$. This figure is in dimensional units with $m$ in atomic mass units and $T$ in seconds 


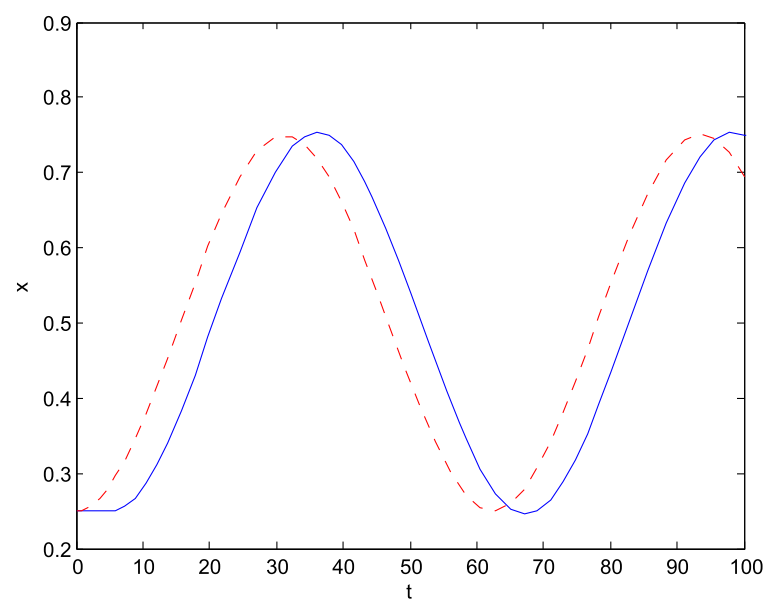

Fig. 8 The full numerical solution to (Eq. 2) plotted in a blue solid curve along with the full numerical solution to (Eq. 9) plotted in a red dashed curve. This figure demonstrates the synchronous period between the two models

Finally, in Fig. 8, we plot the $x(t)$ solution (in blue solid) from the full numeric simulation of (Eq. 2) along with the solution $x(t)$ (in red dashed) from (Eq. 9). The approximation of the integral model ignores the portion of the time spent outside the device, and this has the effect of inducing a phase difference between the predicted and numerical solutions.

The period approximation (Eq. 11) is the exact period for the red dashed solution and an approximation to the full period. Indeed, the figure shows the agreement between the periods of the two models. The phase difference results from model Eq. (9) approximating the system with the mass already inside a one-dimensional oscillator while the full model has a lag as the mass enters the device.

\section{Solenoidal design numerical results}

We include a plot of a trajectory found in the solenoidal model in Fig. 9. With the solenoid, there is minimal bending, and although not evident from the plot, there is a nearly constant drift of the ionized particles within the device instead of a helix as desired. We attribute this to the fact that when the helix is tightly coiled (as is necessary to reduce the

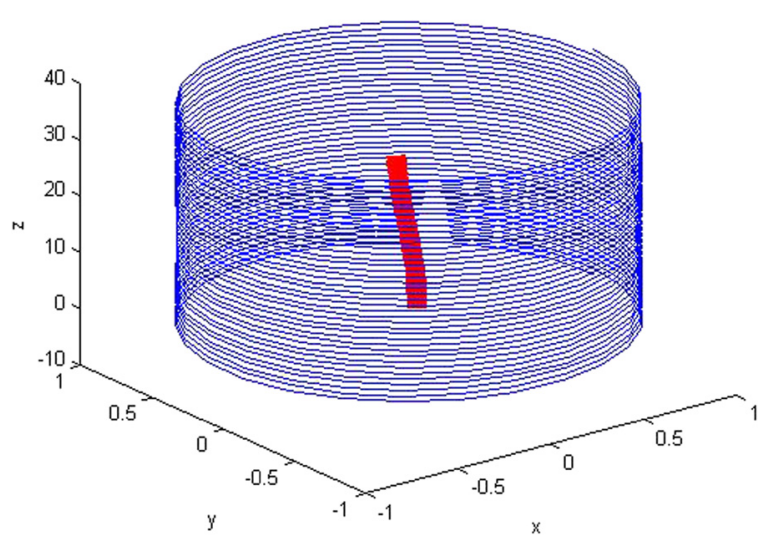

Fig. 9 This plot shows the trajectory of the charged particles (red) entering through the device (blue) at $z=0$. Note there is a slight deflection, but ultimately, there is only a drift 
risk of particles leaving the device through its sides) then a uniform voltage on the helical walls very closely approximates a constant potential on the walls of a cylinder whereby the inner electric field is zero and thus particles drift but are not accelerated.

\section{Conclusions}

We have designed a device that separates components of a chemical substance by mass and detects them simultaneously, thus addressing the problem that was proposed by the industrial partner. Our device solely uses an electric field and does not trap the ions as they travel thus being considerate of both the financial and physical constraints stipulated by the sponsoring company. While the full model is relatively intractable to analytic analysis, we have demonstrated that with some very insightful simplifications, we can recover a mass-dispersion relation for any sample. While actual device imperfections make this curve a theoretical one, it could still serve as a benchmark for calibrating any machine made using this technology.

Possibly by combining a solenoidal design with more complicated engineering practices, such as a length-dependent potential or variable coil radius, the desired effects could be produced.

We decided to not focus on the feasibility of particle detection when considering our model as this is an entirely different engineering problem. Here we propose two methods that could be useful in detecting different types of particles. Firstly, we consider that an areal detector could be placed along the device. Since the particles travel along different trajectories, they would cross an areal detector at different positions in the plane of the detector. Using data fitting to the curve generated in Fig. 7, one could calibrate the device based on certain control masses. A second detection mechanism could involve the ability to measure the oscillating signal of the masses. Through Fourier techniques, the oscillations could be decomposed and the mass constituents identified.

Competing interests

The authors declare that they have no competing interests.

Authors' contributions

This paper was written and edited by ML and IM. Mathematical analysis of the models, numerical simulations of the trajectories, and force interaction simulations were done by $\mathrm{ML}$ and IM. Parts of the particle-particle force interaction code were provided by KR. ML, IM, and KR compiled the references. All authors read and approved the final manuscript.

\section{Acknowledgements}

The authors would like to thank the Fields Institute for hosting the 2014 Fields-MPrime Industrial Problem Solving Workshop that provided us the venue and opportunity for this work and for paying for our travel expenses and accommodations. The authors also wish to thank Jeremy Budd and Mary Pugh for their involvement in the group work at the workshop, Samad Bazargan of PerkinElmer for presenting this very interesting problem, and the referee for their helpful suggestions in revising this paper.

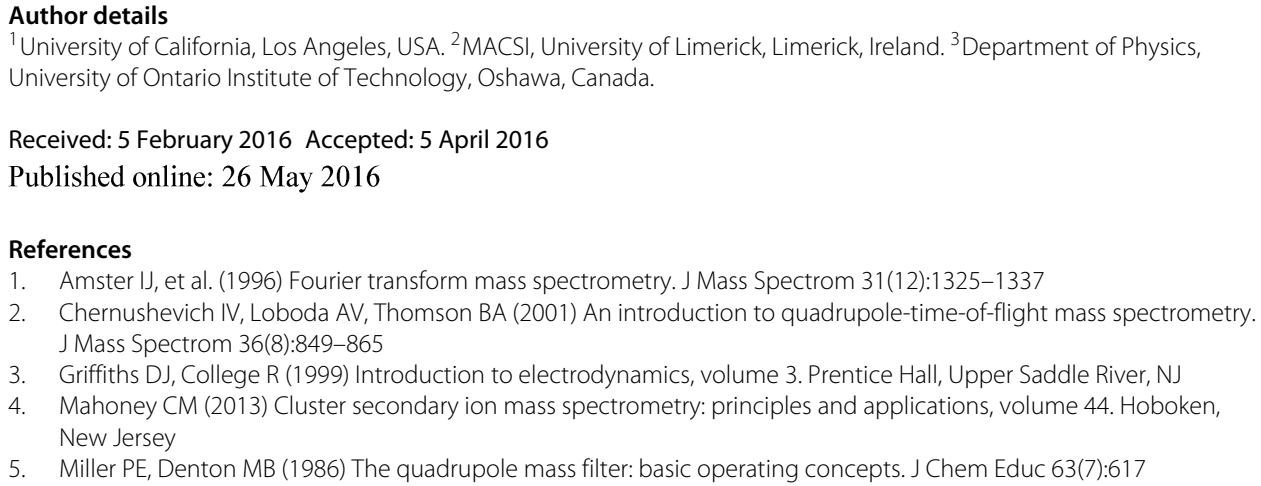

5. Miller PE, Denton MB (1986) The quadrupole mass filter: basic operating concepts. J Chem Educ 63(7):617 